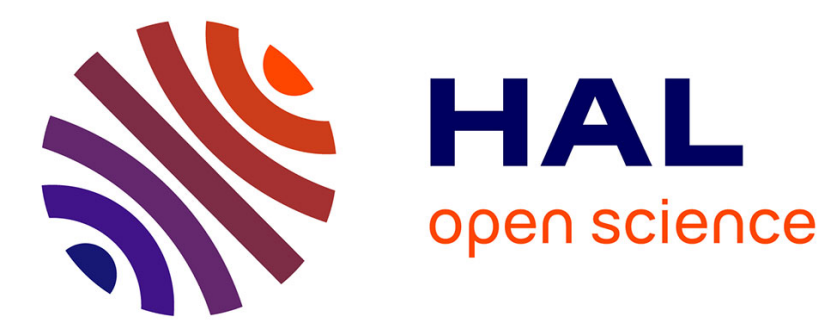

\title{
RAY THEORY OF THERMAL WAVE IMAGING
}

J. Burt

\section{To cite this version:}

J. Burt. RAY THEORY OF THERMAL WAVE IMAGING. Journal de Physique Colloques, 1983, 44

(C6), pp.C6-453-C6-457. 10.1051/jphyscol:1983672 . jpa-00223231

\section{HAL Id: jpa-00223231 https://hal.science/jpa-00223231}

Submitted on 1 Jan 1983

HAL is a multi-disciplinary open access archive for the deposit and dissemination of scientific research documents, whether they are published or not. The documents may come from teaching and research institutions in France or abroad, or from public or private research centers.
L'archive ouverte pluridisciplinaire HAL, est destinée au dépôt et à la diffusion de documents scientifiques de niveau recherche, publiés ou non, émanant des établissements d'enseignement et de recherche français ou étrangers, des laboratoires publics ou privés. 


\title{
RAY THEORY OF THERMAL WAVE IMAGING
}

\author{
J.A. Burt \\ Department of physics, York University, Toronto M3e 1P3, Canada
}

\begin{abstract}
Résumé - On applique la théorie des rayons à l'étude d'une lame de rasoir par onde thermique. Un bon accord est obtenu pour le champ lointain lorsque $1^{\prime}$ on tient compte de la loi de Boltzmann du corps noir. L'effet de la variation de la conductivité thermique avec la température est très faible.
\end{abstract}

Abstract - The thermal ray theory is applied to the experimental thermal scan on a razor blade. Good magnitude agreement is obtained using a far-field ray formula provided correction is made for the Boltzmann blackbody radiation law. The effect of conductivity variation with temperature is found to be small.

\section{Introduction}

If a modulated heat source produces thermal waves in a sample then the resulting modulated heat diffusion exhibits ray properties and the ray may be considered to reflect, refract and diffract just as acoustical rays. As an illustrative example we apply the ray theory to Busse's [1] thermal wave apparatus. The theory is not restricted to his technique, however, but will describe thermal waves in any situation. In Busse's technique a modulated CW laser beam is incident on the front surface of an opaque sample and a sensitive IR detector is focussed to a point opposite the laser beam on the rear surface. The blackbody radiation from the rear surface focussing point depends on the thermal.path from the laser incidence point on the front surface to the detection point on the rear surface. Any subsurface structure within the sample such as holes or inclusions will influence the final temperature detected. If, then, the detector is mechanically scanned during the measurement, a line or one-dimensional image of the sample structure is obtained. To a first approximation, this line image is integrative in sample depth but not, as is the case in a sealed photoacoustic cel1, integrative over the scanned surface since only a single point contributes to the signal rather than the entire rear surface. Consequently better spatial resolution is obtained using Busse's technique than in the photoacoustic case.

Any theoretical description of the temperature within the sample (and finally at the detection point on the rear surface) must at least be two-dimensional. Since few analytic methods are available for solving two-dimensional heat conduction it is worthwhile to consider a ray technique based on Keller's [2] optical diffraction ray theory as it promises to involve less computation than a purely numerical integration such as the finite element method. It is well known that modulated thermal waves are rapidly attenuated with distance. Effectively this means that few rays need to be considered since many would travel such long distances before arriving at the detection point that they contribute virtually nothing to the total signal. Thus, unlike optical rays where only problems including diffraction are practicable, for the thermal rays (or rays in any lossy medium) solutions may be obtained even if nothing more than reflection is present since few rays need be calculated. Such is the case for the example under study in this report. We point out that for the photoacoustic case, there is yet another technique which does not involve the lengthy computation of numerical integration yet is nevertheless applicable to a variety of sample structures namely the Green's function method of Thomas et a1. [3]. Due to the difficulty in finding Green's functions for complicated geometrical boundaries, the method is limited in the type of sample subsurface structure that can be 
considered. The ray method, however, is not restricted to any type of subsurface geometry.

Our analysis begins with the Fourier heat conduction law:

$$
\nabla^{2} \mathrm{~T}-\frac{\mathrm{I}}{\alpha} \frac{\partial \mathrm{T}}{\partial \mathrm{t}}=\frac{\mathrm{Q}}{\mathrm{k}}
$$

$T$ represents the temperature, $t$ the time, $\alpha$ the thermal diffusivity, $k$ the thermal conductivity, and $Q$ the energy flux. For a real, modulated heat source, $Q$ is always positive so that the temperature, even with modulations, always decreases away from the source. If we assume that the time varies as $\exp (-i \omega t)$, $\omega$ bieing the angular frequency, then the Helmholtz equation results:

$$
\nabla^{2}+\frac{i \omega}{\alpha} \mathrm{T}=\frac{\mathrm{Q}}{\mathrm{k}}
$$

with wavenumber equal to $\sqrt{i \omega / \alpha}$, i.e., the roots of the characteristic equation have real and imaginary parts. The real part describes the attenuation along the ray. The fact that equation (2) is a Helmholtz equation implies that the thermal rays will reflect, refract and possess the usual reflection and transmission coefficients at media interfaces, i.e., at the boundaries of the sample. The thermal rays, however, are not identical to the solutions of the Rosencwaig-Gersho theory [4] which for the case of a plane slab with parallel faces excited by a planar heat source shows only two waves, a forward and a backward travelling one. Calculating the thermal rays involves a large number of reflections since all rays are normal to the parallel faces. In this case, then, the ray solution presents no advantage whatsoever since many waves must be considered rather than the two of the Rosencwaig-Gersho theory. If, however, the source illumination is focussed to a point then the RosencwaigGersho theory no longer applies since the thermal wavefront is not planar and one seeks instead a spherical wave solution which may be obtained using thermal rays.

Two problems in utilising thermal ray analysis are: (1) the algebraic representation to.be used for the ray magnitude and phase, and (2) determining the number of possible types of rays and devising algorithms for their computation. Concerning the second problem, we note that only two algorithms are necessary for the solid sample of this study: one for the ray proceeding directly from the source point to the detection point and one for rays reflecting any number of times before reaching the detection point. We note that ray types must be determined by trial and error and this becomes a serious problem in more complicated geometries. For example, the same sample considered here but with a subsurface hole [6] would require as many as nine different ray types.

The algebraic representation for the thermal ray need not be a single one since the thermal waves travel through both the near and intermediate radiation zone before severe attenuation. Using experimental agreement to guide us, the algebraic representation we chose was a single one, for simplicity, and moreover it was the asymptotic far-field form. Keller's results show that this form is also a reasonable approximation in the intermediate zone. The representation chosen was: $\mathrm{T}=1 / \mathrm{r} \exp (-\mathrm{r} / \mathrm{I})$,

where $r$ is the path length and 1 the thermal diffusion length.

We have reported previously the principle of the thermal ray method [5], and its application to a sample containing a single hole [6]. The present example considers Busse's experimental data for a thermal scan along a razor blade. Since the sample is much thinner than the thermal diffusion length, the role of reflections should be enhanced. Additionally, the $4 \mathrm{~W}$ argon ion laser focussed on such a thin sample can cause temperatures high enough that a Boltzmann's radiation law correction must be applied to the temperature detected. Further, since the sample is iron, the thermal conductivity will change considerably with the induced temperatures.

Experiment and Discussion

The representation (3) is for the three-dimensional case, but we view the very broad sample on its edge so out-of-plane waves will never return to the solution plane. One difficulty is that this far-field representation is, of course, singular at 
zero $r$. How small $r$ may be with (3) still valid depends on the modulation frequency and the diffusivity. This is seen in figure 1 which shows only the thermal wave magnitude on two different scales as a function of $r$, the radial distance through the sample. The exact solution for a $20 \mathrm{~Hz}$ thermal wave in an infinite iron medium [7] is presented along with the ray theory calculation. Since the medium is infinite there are no reflections to consider. The ray representation was further divided by the factor $1+\exp (-\mathrm{hr})$, with $\mathrm{h}=5 \times 10^{5}$. This gave a slight improvement in the fit for the smallest $r$ values but because of the large value of the exponent the influence of this empirical factor for other $r$ values was very small. In the figures, each magnitude curve was normalized to its maximum and the two curves plotted together at the right-hand end point so that the asymptotic behaviour may be seen. Figure 1 shows that, for the smallest numerical step which we considered, the far-field spherical wave formulation does not deviate greatly from the exact analytic form in modulation magnitude.
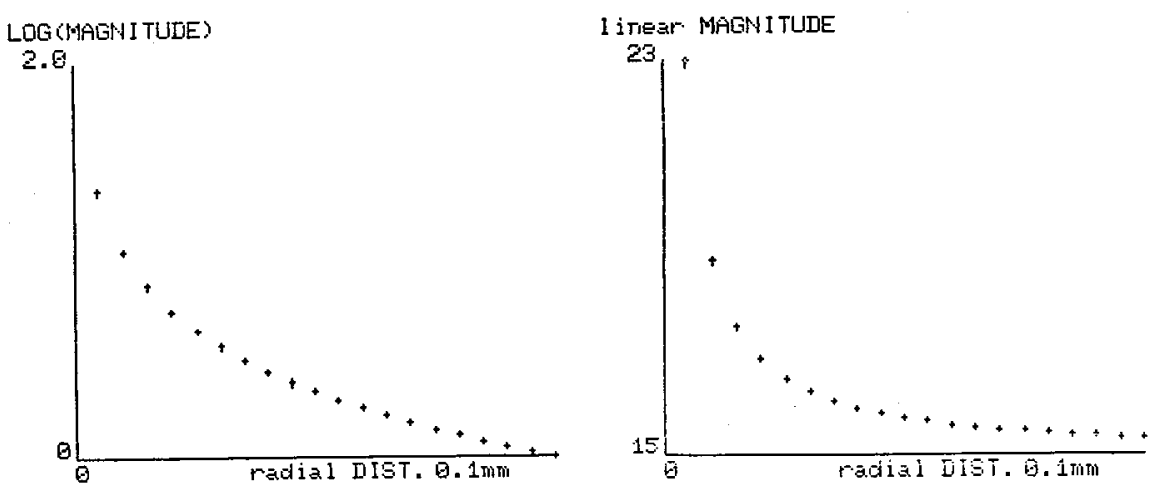

Fig. 1 Logarithmic and linear magnitude of modulated thermal waves vs. radial distance calculated by analytic method (bars) and ray method (stars).

Since the sample material is iron whose conductivity varies with temperature, hence the conductivity varies with distance throughout the sample. In a trial calculation., temperatures were evaluated inside the sample using the thermal ray formulation and at each radial increment the conductivity was changed. Virtually no difference was found between the temperatures so calculated and those obtained by using a single ray representation based on the room temperature conductivity.

Busse's experimental thermal scan of an $0.1 \mathrm{~mm}$ thick razor blade is shown as the barred points in Figure 2. The distance the detector is scanned along the sample back face is shown on the $\mathrm{X}$-axis as SCAN. The magnitude of the $20 \mathrm{~Hz}$ thermal scan signal extends over nearly two log decades. We calculated a direct ray from the laser incidence point to the current scan detection point on the back surface of the sample, and added to it 15 multiple reflections having the same initial and final points. The result is shown in Figure 2 as the starred curve where the calculated phase is shifted to coincide with the experimental at the far right point. The agreement with experiment is neither good in the asymptotic region at a scan distance of $1.2 \mathrm{~mm}$ nor at small scans.

Finally, the thermal emission was corrected by the Boltzmann radiation law. This correction was applied to the magnitude on $1 \mathrm{y}$ and the result in Figure 3 shows much improved agreement. An initial spot temperature on the front of the razor blade was assumed and the remaining points calculated using the ray procedure. The fit 
with experiment depended on the initial laser-focussed spot temperature assumed. When the best fit was found at spot temperature $700 \mathrm{~K}$, this result was compared with an analytic calculation for a pulsed heat source [7] using a pulse length equal to one half the $20 \mathrm{~Hz}$ period.
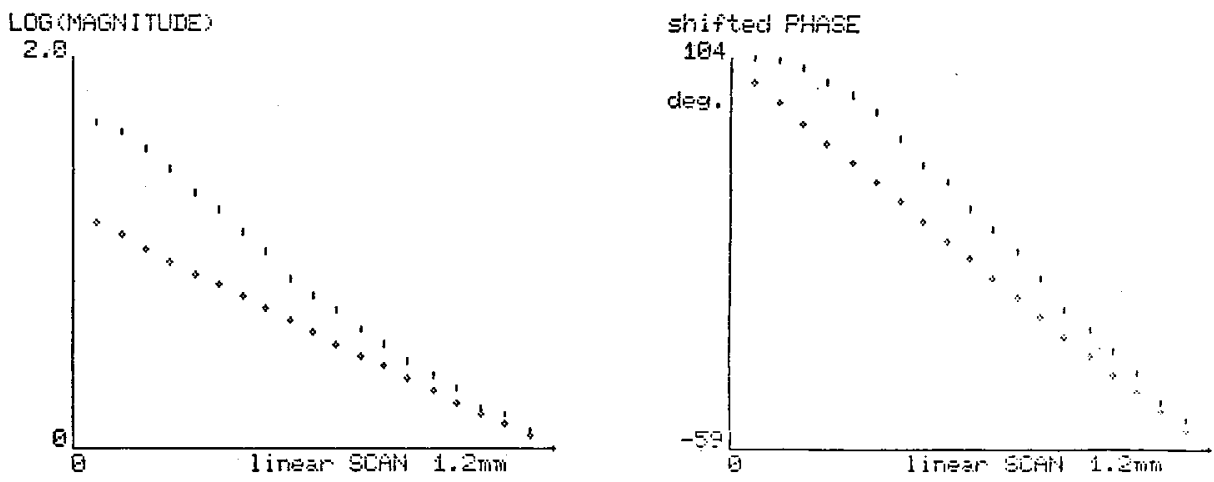

Fig. 2 Thermal scan of razor blade. Experiment (bars), and theory with direct and reflected rays (stars).
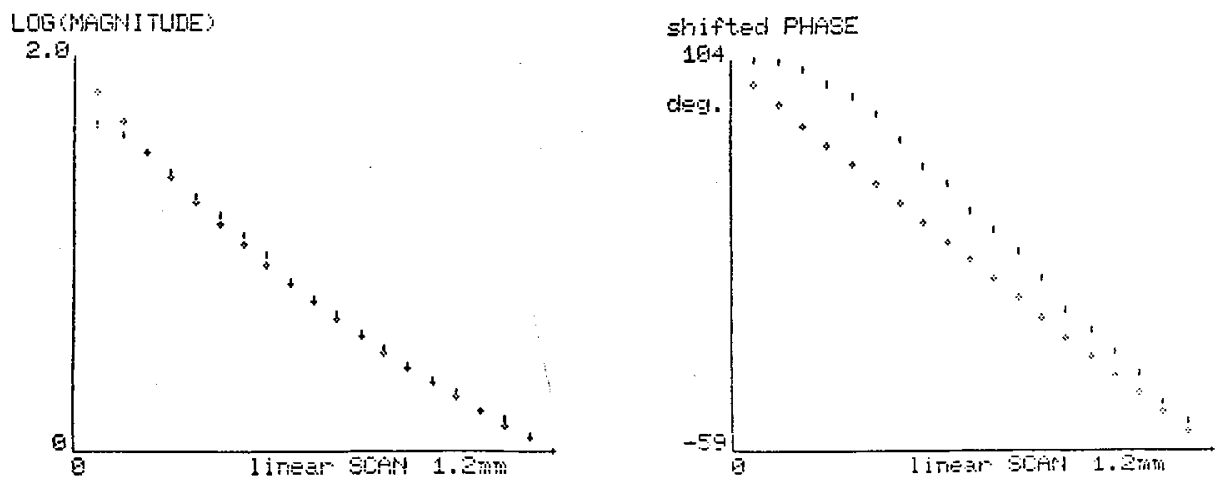

Fig. 3. Thermal scan of razor blade. Experiment (bars), and theory with direct, reflected rays, Boltzmann radiation correction on magnitude (stars).

This is only an approximation to the steady periodic solution but the agreement obtained is significant, namely, for a spot radius of 10 micrometres and laser power 1.7 watts the front surface spot temperature expected is $690 \mathrm{~K}$. This spot radius and power agrees with experiment. No correction has been applied to the phase curve so the experimental and theoretical phase remain in disagreement. 
In conclusion, we have illustrated some of the considerations necessary when applying ray theory to thermal waves in a particular experimental example. One ultimate objective of analysis by ray theory is to provide the basis for computer holographic image processing of the experimental data so that sample subsurface structure may be brought into focus.

Acknowledgement

The writer acknowledges his debt to Dr. G. Busse for his generosity in providing the experimental data and for his continuing encouragement and constructive criticism. The writer also gratefully acknowledges the support of NSERC, Canada, in the form of an Operating Grant.

\section{References}

[1] BUSSE G., Infrared Phys. 20 (1980) 419.

[2] KELLER J.B., J. Opt. Soc. Amer. 52 (1962) 116.

[3] THOMAS R.L., POUCH, J.J., WONG, Y..H., FAVRO, L.D., KUO, P.K., ROSENCWAIG, A., J. App1. Phys. 51 (1980) 1152 .

[4] ROSENCWAIG, A., GERSHO, A., J. App1. Phys. 47 (1976) 64.

[5] BURT J.A., Second Conference on Photoacoustic Spectroscopy, (1981).

[6] BURT J.A., IEEE U1trasonics Symposium, (1981).

[7] CARSIAW, H.S. and JAEGER, J.C., Conduction of Heat in Solids, (Oxford) 1973.

[8] ARECCHI, F.T. and SCHULZ-DUBOIS, E.0., (Ed.) Laser Handbook, v.2, p.1587, (North-Holland, Amsterdam) 1972. 